\title{
Implementing a Cooking and Dietary Management System Using RFID Technology
}

\author{
Pai-Hsun Chen, Ying-Hsin Liang, and Tsung-Chi Lin \\ Department of Multimedia Animation and Application, Nan Kai University of Technology, 568 Chung Cheng Road, \\ Caotun Township, Nan Tou County 54243, Taiwan
}

Correspondence should be addressed to Ying-Hsin Liang; t136@nkut.edu.tw

Received 25 February 2014; Accepted 4 April 2014; Published 7 July 2014

Academic Editor: Her-Terng Yau

Copyright (c) 2014 Pai-Hsun Chen et al. This is an open access article distributed under the Creative Commons Attribution License, which permits unrestricted use, distribution, and reproduction in any medium, provided the original work is properly cited.

\begin{abstract}
The establishment of a database of nutritional ingredients with electronic scales feature provides chefs with conducted cooking; as long as the food category is selected and weighed by order, the nutritional content of the dish will be known. By RFID electronic scales and electronic plate function, the meal intake of nutrients and measurement of food nutrients per meal can reach a daily diet control purpose. The so-called RFID electronic plate is embedded into the chassis and aplate composed of a plurality of dishes to detect information in the chassis and the various subdishes through RFID reader. The chassis provides users with automatic identification such as physiological signals and doctor's prescription as an individual dietary recommendation through dietary database. The dietary database, in addition to providing essential food nutrients, can be used to query using keywords and classification methods so as to quickly find the sum ingredients for cooking and ingredients. The chefs instantly know the meals nutrition during ingredients weighing by using RFID electronic scales. End users only need to place the allocated cooking dishes on electronic scales; they can easily know the total own meal intake nutrition.
\end{abstract}

\section{Introduction}

Busy lives affect changing in eating habits. Due to increased eating out population, the general public tends to be highfat diet, low-fiber foods, lack of exercise plus static lifestyle, which results in obesity and nutritional imbalance, such as the daily excess calorie intake and the insufficient protein intake, and thus bodily functions change, such as body fat increase and muscle loss leading to reduced muscle mass, muscle strength falling, and a decline in physical function, and chronic diseases will produce derivatives. It begins to rise in the long-term care family structure and function of the human potential future demanding pressures. The American Society for Parenteral and Enteral Nutrition defines, "Malnutrition is any kind of nutritional status of obstacle, including the lack of nutritional intake, nutritional and metabolic deficiencies or excess nutrients circumstances." Therefore the usual dietary intake and then right to moderate exercise is discretionary health fundamental. Bandura and Johnson pointed out that malnutrition increases the risk of disease and death struck probability, which will lead to social costs and burden of medical resources $[1,2]$. Therefore, proper assessment and understanding of the daily nutritional intake conditions, early detection, and timely intervention of eating problems will be great help for mastering to maintain a healthy nutritional status and lower medical expenses.

The nutrition screening initiative (NSI) estimated that $20-60 \%$ of elderly people have problems at home or are at high risk of malnutrition. According to the Department of Health, lack of dietary fiber can cause not enough gastrointestinal motility and constipation problems to affect intestinal health. Excessive intake of cholesterol can cause cardiovascular disease and increase risk of heart disease. Excessive heat is likely to cause obesity, but the lack of calorie intake will cause lack of energy and abnormal body metabolism. Lack of protein will prone to stunting, easy fatigue, weakened immunity, and so on. Due to the high blood sugar, high cholesterol, hypertension, and cardiovascular disease, there are important factors for cerebrovascular disease progression. In view of this, the Department of Health will put high blood sugar, high cholesterol, and high blood pressure control as an important nation work among control programs of 
geriatric health plan. Whatever the nutrients are, excessive intake or too little intake will produce health problems. And diet management system is nothing more than to explore the physiological data for users to analyze, and the user is required to calculate the daily nutrition, good nutrition under intake to reach a healthy diet purposes [3-5].

The rest of the study is organized as follows: Section 2 presents a brief review of trellis-based informed embedding in diet and disease; Section 3 provides a description of our major work on research methods; Section 4 provides results; Section 5 provides a discussion; finally, Section 6 offers conclusions.

\section{Related Works}

Proper diet and moderate exercise are the only way to maintain good health. However, people often are not very concerned with diet control, and it causes some health problems. The effective ways to remain healthy is by controlling diet and doing exercise. Therefore, the understanding of their diet ingredient is an important issue. Firstly, we will recognize a dish feature which is proved from "My plate" by embedding an eTag. The identification function of eTag can record intake of nutrients at each meal for different users and according to their needs to make different suggestion to achieve customized demand.

2.1. Proved "My Plate". Taiwanese home cooking habits: people access their meal which is according to their own preferences to take dishes and amount; unknowingly, they may pick up excess food or unbalanced diet. So, before exploring the relationship between disease and diet, to design a diet plate for Taiwanese is necessary.

As we know, in 1992, "food pyramid" was first issued by the United States. The food pyramid is classified systematically to show the nutritious food for people. However, owing to the differences in age, gender, and physical condition, the "food pyramid" is hard to operate actually for daily diet. Therefore, the Ministry of Agriculture has decided to completely abandon the "pyramid" to enable the new concept. The Secretary of the United States Department of Agriculture, Tom Vilsack, said that the food pyramid cannot be easily and quickly used in general by the American family. He published "My Plate" which is the new label to demonstrate the daily diet concepts [6]. "My Plate" is divided into four different color blocks: the red block points out fruits requirement, the green zone is for vegetables demand, orange is for protein, and purple is for cereal. In addition, next to the plate placed in a blue area is figured diary milk products demand. "My Plate," which was shown in Figure 1, has been used to replace food pyramid in the U.S. and has become the new graphic for U.S. healthy eating guidelines $[6,7]$.

(1) Revised E-Tag Plate of "My Plate." The concept of universal design is the first stages of design process, it points out the general needs of average people. Its design steps are without special consideration so that it does not need to spend more time and a lot of money to develop its prototype, and the

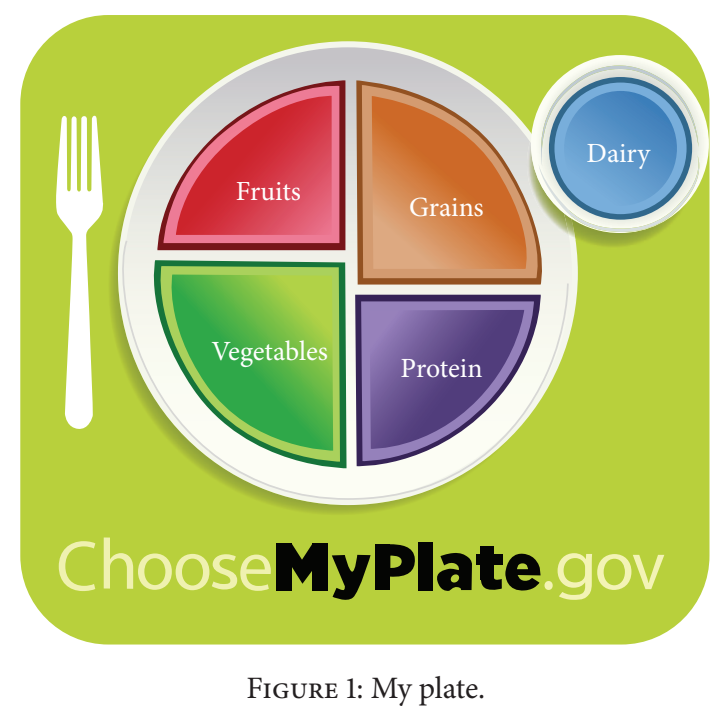

finished product cannot be afforded for special customers. Therefore, to enhance the value of universal design is an important issue. If the concept of universal design which can be combined into the design process and its products can better meet the needs of most users, it can achieve the scale of the market economy [8].

For the convenience of proving "My Plate" structure to combine the individual dishes with embedded RFID tag to match Taiwanese home cooking habits, they always access their own dishes and amount depending on the preferences of the family members. This study incorporates IT technology to produce a dietary management system. According to the function of identification (RFID) tag, added electronic tags with personal health management information, configuration of nutrients, and food information of the plate, food ingredients, and diet calorie management information. By obtained meal information of the plate, diet calorie configuration of nutrients can be achieved to make dietary management more convenient to match Taiwanese needs.

To achieve the above idea, e-Tag plate designed features are divided into "chassis" and "combination plate."

(i) The "chassis" is the base to contain each combination plate, which is embedded with RFID-Tag to record personal health management information.

(ii) The "combination plate" is the set to be used for containing dishes and is also embedded with RFIDTag. Based on database of calories and nutrients information, through the weighting information, the RFID-Tag of chassis is combined to count out the calorie and present total calories and nutrients contained in the dishes. The improved of "My Plate" is shown in Figure 2.

(iii) To facilitate picking up and cleaning of the combination set of plates, the chassis is designed with a recess in compliance with the shape and size of the finger. The grip plate is shown in Figure 3. 


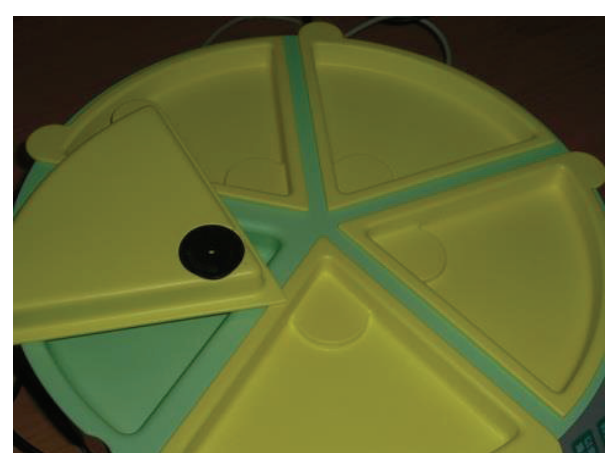

FIgURE 2: Revised plate embedded RFID tag.

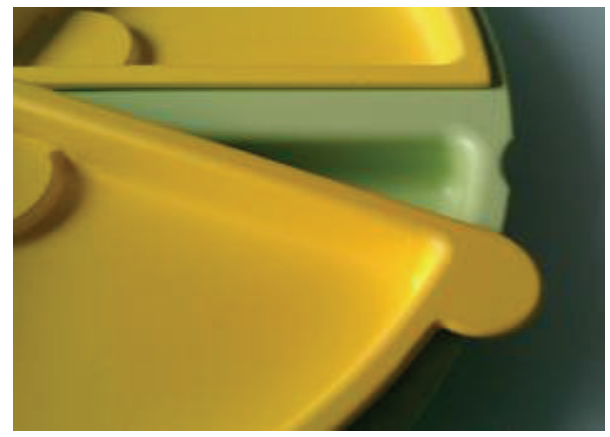

FIGURE 3: The grip plate.

(iv) In order to read food nutrients while postcooking dishes are configured by using RFID, the modified RFID electronic scale needs to be built to read RFIDTag data; therefore, the amount of food nutrition will be shown for each combination plate of food; users can easily know the sum of the food intake of nutrients of their own. The modified RFID electronic scale is shown in Figure 4.

(2) Consideration of RFID Technology. In order to value "My Plate," this study proposes a method to improve the plate, which can be a healthy diet management system embedded with IT technology. RFID technology is already a very mature electronic technology, which is commonly used in manufacturing, selling, library management, identification, supply chain management, and so forth. It can save cost of labor management and data processing. Therefore, it could be a very exciting topic to bring the benefits of RFID to the catering trade [9].

The reasons why this study takes RFID as an identified system are represented as follows.

(1) There is not any limit to the number of times used to add, modify, and delete data stored in the RFID tag.

(2) It does not need to be close to the object, just within the RFID tag radio range detected.

(3) Tags have bigger data storage capacity than barcodes.

(4) Tags can be used repeatedly.

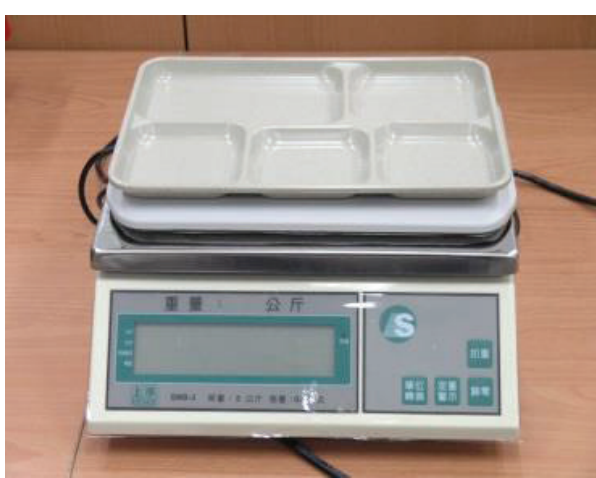

FIgURE 4: The modified RFID electronic scale.

(5) The identification can be carried simultaneously on multiple tags at the same time.

(6) RFID read/write is password-protected, so high security protection makes it difficult to be forged and altered.

This research uses the RFID security to improve my plate. AES (advanced encryption standard) is applied as security protection, which is used to replace the original DES (data encryption standard), and has been widely used around the world. The advantages of AES analysis are high speed and simple but tough algebraic computation and are difficult to decipher, so it can quickly add decryption algorithms. For the AES-128 algorithm, the length of the cipher key is 128; the key length is represented by $N_{k}=4$, the number of rounds is represented by $N_{r}=10$, and block size is represented by $N_{b}=4[10]$.

AES encryption process is based on a $4 \times 4$ matrix operation; there is a state (block) as an element of matrix. And the loop of each encryption round contains four steps:

(1) AddRoundKey: key and data make XOR operation, which is $b[i, j]=a[i, j]$ Xor $k[i, j]$.

(2) SubBytes: each byte should be converted into $S$ matrix through $S$-Box function (using table look-up); that is, $b[i, j]=S(a[i, j])$.

(3) ShiftRows: each row of the matrix should be carried for a cyclic shift, and a shift step is proportional to the size of the column number.

(4) MixColumns: each column of matrix should be multiplied by a polynomial.

When running $N_{r}$ rounds of encryption codes, from $N_{r}-1$ rounds, each round sequentially executes SubByte (), ShiftRow (), MixColumn (), and AddRoundKey () operations. And then the $N_{r}$ rounds perform sequentially ByteSub (), ShiftRow (), and AddRoundKey () operations. In order to make all bytes in the AES algorithm interpreted as finite field elements, there is a structure of the finite field GF $\left(2^{8}\right)$ which is the set of 256 possible byte values converged in a finite domain by XOR operation. AES encryption processes are described as below. 




Figure 5: Applying the $S$-box to each byte of the state for SubBytes () mapping.

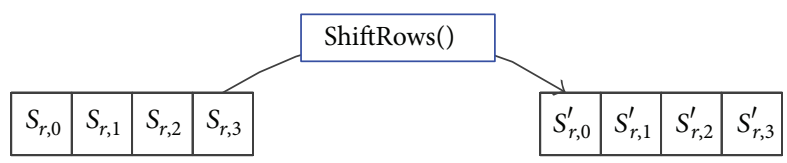

FIGURE 6: Illustrating the ShiftRows() transformation.

(i) SubBytes() Transformation. S-box consists of a GF $\left(2^{8}\right)$ used to convert each block of bytes. In a mapping form, the SubBytes() transformation of the $S$-box can be expressed as in Figure 5.

(ii) ShiftRows() Transformation. The shift value of shift $\left(r, N_{b}\right)$ depends on the row number $r$. If $N_{b}=4$, shift $(1,4)=1$, shift $(2,4)=2$, and shift $(3,4)=3$. The diagram of ShiftRows () transformation is shown in Figure 6.

(iii) MixColumns() Transformation. To avoid the columns of state over GF $\left(2^{8}\right)$ polynomial, it considers the multiplied polynomial $a(x)=\{03\} \times 3+\{01\} \times 2+\{01\} x+\{02\}$ and modulo $(x 4+1)$. The equation is presented as $s^{\prime}(x)=a(x) \oplus s(x)$, and the matrix form is shown below, and the result of this multiplication is replaced by the following equation which illustrates the MixColumns() transformation:

$$
\begin{aligned}
{\left[\begin{array}{c}
s_{0, c}^{\prime} \\
s_{1, c}^{\prime} \\
s_{2, c}^{\prime} \\
s_{3, c}^{\prime}
\end{array}\right]=\left[\begin{array}{llll}
02 & 03 & 01 & 01 \\
01 & 02 & 03 & 01 \\
01 & 01 & 02 & 03 \\
03 & 01 & 01 & 02
\end{array}\right]\left[\begin{array}{c}
s_{0, c} \\
s_{1, c} \\
s_{2, c} \\
s_{3, c}
\end{array}\right] \quad \text { for } 0 \leq c \leq N_{b}, } \\
S_{0, c}^{\prime}=\left(\{02\} \cdot S_{0, c}\right) \oplus\left(\{03\} \cdot S_{1, c}\right) \oplus S_{2, c} \oplus S_{3, c}, \\
S_{1, c}^{\prime}=S_{0, c} \oplus\left(\{02\} \cdot S_{1, c}\right) \oplus\left(\{03\} \cdot S_{2, c}\right) \oplus S_{3, c}, \\
S_{2, c}^{\prime}=S_{0, c} \oplus S_{1, c} \oplus\left(\{02\} \cdot S_{2, c}\right) \oplus\left(\{03\} \cdot S_{3, c}\right) \\
S_{3, c}^{\prime}=\left(\{03\} \cdot S_{0, c}\right) \oplus S_{1, c} \oplus S_{2, c} \oplus\left(\{02\} \cdot S_{3, c}\right)
\end{aligned}
$$

(iv) AddRoundKey() Transformation. This transformation is presented by making a XOR operation of key and state, and the equation is shown as $s^{\prime}[i, j]=s[i, j]$ Xor $w[i, j]$ :

$$
\begin{aligned}
& {\left[S_{0, c}^{\prime}, S_{1, c}^{\prime}, S_{2, c}^{\prime}, S_{3, c}^{\prime}\right]} \\
& \quad=\left[\begin{array}{llll}
S_{0, c}, & S_{1, c}, & S_{2, c}, & S_{3, c}
\end{array}\right] \oplus\left[w_{\text {round } * N_{b}+c}\right] \quad \text { for } 0 \leq c<N_{b} .
\end{aligned}
$$

The decryption algorithm of AES is executed by a reverse pseudocode, which contains three reverse functions: InvShiftRow(), InvByteSub(), and InvMixColumn().

The advantages of AES operation are that all operators can be converted into an exclusive OR operation and a lookup table, which is efficient as well as previously described. Encoding decoding free especially is a free encryption and encryption tool, which provides Codifica AES 123-bit encryption technology. The main encryption program is not only safe but also quick and easy to currently operate with dragging a mouse to achieve encryption and decryption.

2.2. The Association between Diet and Disease. The causes of morbidity or disability originate in normal aging and first body organs degradation, including the kidneys, heart, and lungs, as well as chewing, digestion, and absorption functions. These functions will gradually decline with age, and secondly these are because of many cumulative sums of risk factors such as blood fats, high blood sugar, and high blood pressure. Therefore, food intake of nutrients and the impact of the disease exists the certain extent of relationship. The cause of chronic diseases and recommended items relative to the diet proposed are shown in Table $1[11,12]$.

Almost all the different chronic diseases need to avoid intake of high-fat, high-calorie, and high sodium content of foods. Avoiding poor eating habits, dietary planning of nutritionist, and good exercise habits can slow the progression of chronic diseases.

In recent years, there are a lot of researches on diet management system, and more of them are general to longterm care facility residents with chronic diseases in order to institutional catering management systems mainly use record daily dietary intake as well as the user's physical condition to use by administration a daily diet. However, the general residents in home with chronic diseases, which are not like the residents in institutional care centers, can get good nutrition care. Thus, since 2001, the Department of Health began promoting regional teaching hospital to hand over discharge planning services to out of hospital diet care services. For example, discharged patients based on conditions are transferred to nutritionist for nutritional care and social works' room services delivery, so that the patient and their families after leaving the hospital can also get food care services.

The most basic control method is to record daily diet intake of dietary nutrients. In recent years, the related diet management system was such as that by Li who proposed a "RFID recorded diet in the application of home health care" [13] and Chang who developed a "diet sensing table-wisdom plane of sensing eating behavior" [14] and so on. Mainly, food nutrition measurements are carried out through RFID identification function and identification of the identity of the meal to control home dietary nutrients. Yao and Wang proposed to "use the principles of design and explore personal diet management system interface" to design for eatingout clan, who simply select the date to which restaurant to eat something so that he can easily record his own diet information [15]. Miyazaki et al. developed "Image-based 


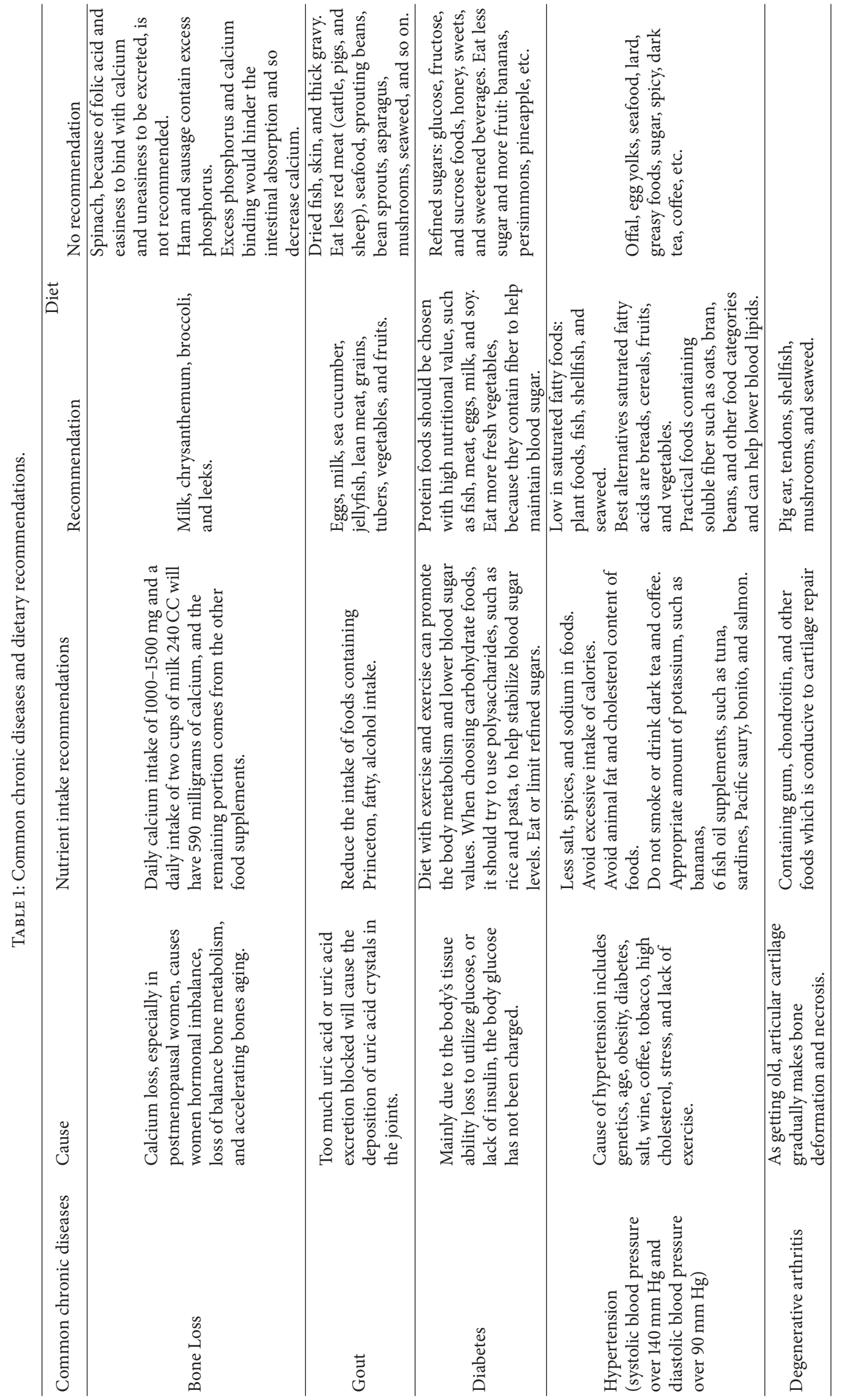


calorie content estimation for dietary assessment" system by using image analysis methods for dietary assessment, nutrition with images, and dietary data record for the diet control and analysis [16]. Kovásznai produced "Developing an expert system for diet recommendation," with the user's dietary restrictions to the diet plan to achieve the purpose of a healthy diet [17]. Mariappan et al. proposed "Personal Dietary Assessment Using Mobile Devices" to record and manage adolescent's diet, which are combined with imaging techniques to improve the record accuracy [18].

However, the average family cooking with different dietary habits makes eating Chinese food at home not easy to manage the use of intervention analysis from cooking to serve and the nutrients of each meal intake. Therefore, this study developed a home-style cooking and catering management system, mainly importing radio frequency identification (RFID) technology to garnish the dishes before cooking process so that nutrients of dishes can be calculated. By the weight of that food intake, home users can ingest nutrients for each meal so as to control the family diet easily.

To achieve the above purposes, this study first constructs a dietary database, where the library stores the user's basic physiological health information and ingredients. Nutrition recipes tables provide garnish dishes process through the use of electronic scales to control nutrients; furthermore, in this study, RFID was embedded into electronic plate composed of chassis and a plurality of dishes. With the functionality of electronic scales and electronic plate of RFID, measuring the food nutrients per channel can reach daily diet control purpose.

\section{Research Methods}

To effectively construct customized cooking and meal management system, the institute provides research methods described below [19].

3.1. System Analysis and Planning. In order to accomplish the first systematic analysis of the contents of the collection plan on human health effects of nutrients, this study interviewed with nutritionists to learn how to perform dietary analysis.

In addition to the nutritional information and ingredients, recipes and diet information were imported into the database to create a dietary database.

Electronic scales and electronic dishes are combined with RFID technology to design RFID system tray food nutrition for providing culinary and dietary management in line with customization needs.

3.2. Research Process. The research process is divided into three stages as shown in Figure 7.

The first phase is to collect a variety of ingredients nutrients and interview with nutritionists to understand the dietary management in clinical implementation circumstances and the problems noted when information were imported into dietary management.

The second phase proposed the diet management system to import diet and catering information ingredients of recipes

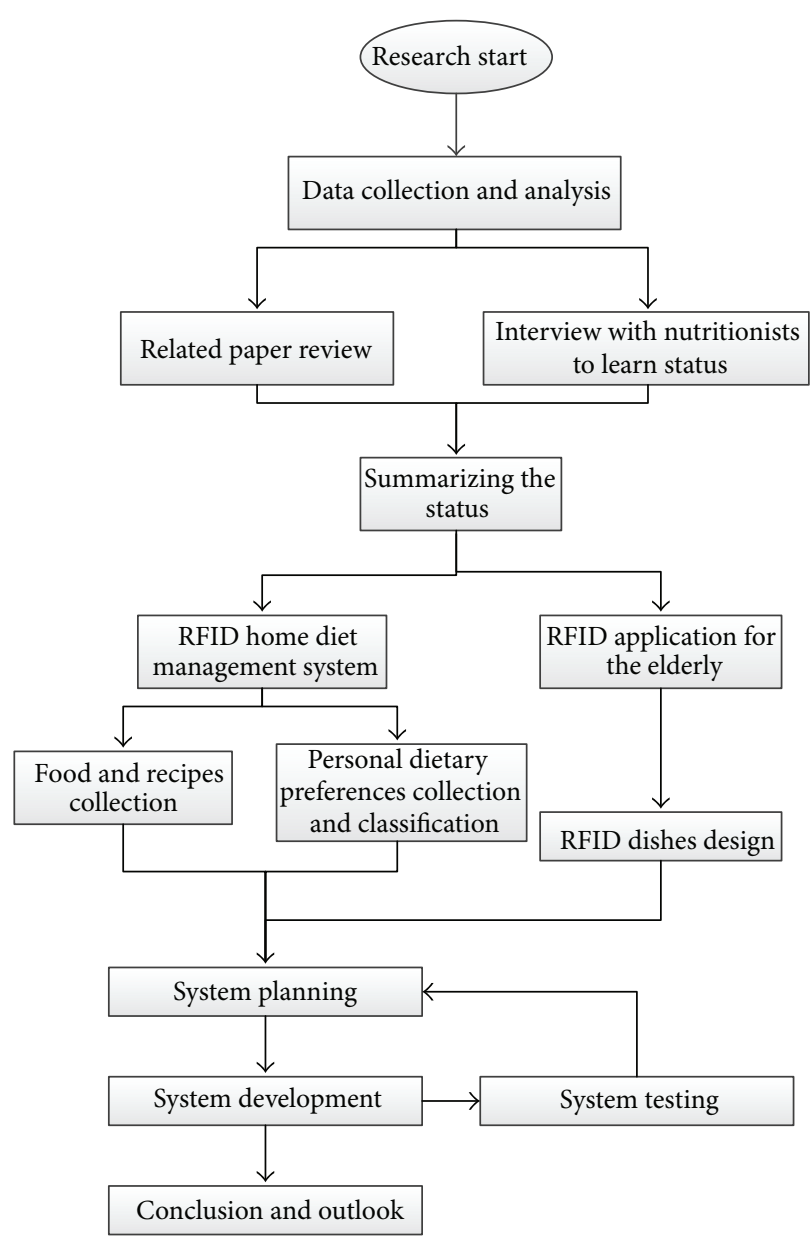

Figure 7: Research flow chart.

into the database to establish dietary database. By electronic scales and electronic dishes integrated with RFID, RFID food tray dish nutrition management system was designed to reach food systems management mechanism.

The third phase, in accordance with the functional requirements of the system planning, conducts writing software, hardware integration, database design, and programming of the system after the completion of the online test.

3.3. System Functions Definition. In this study, RFID technology is used to construct cooking food management system by using functions of ingredients database to establish dietary and nutritional food ingredients, in addition to the application of RFID dishes to calculate dietary nutrients and build personalized measuring physiological information database to provide customized catering services management.

According to the needs, several functions of the following system are summarized to show the relationship between user and system functions.

(1) Dietary care platform: it provides additional nutritional status and personalized menu table ingredients, recipes, menus, preferences, as well as a personalized for delete, modify, and query functions. 


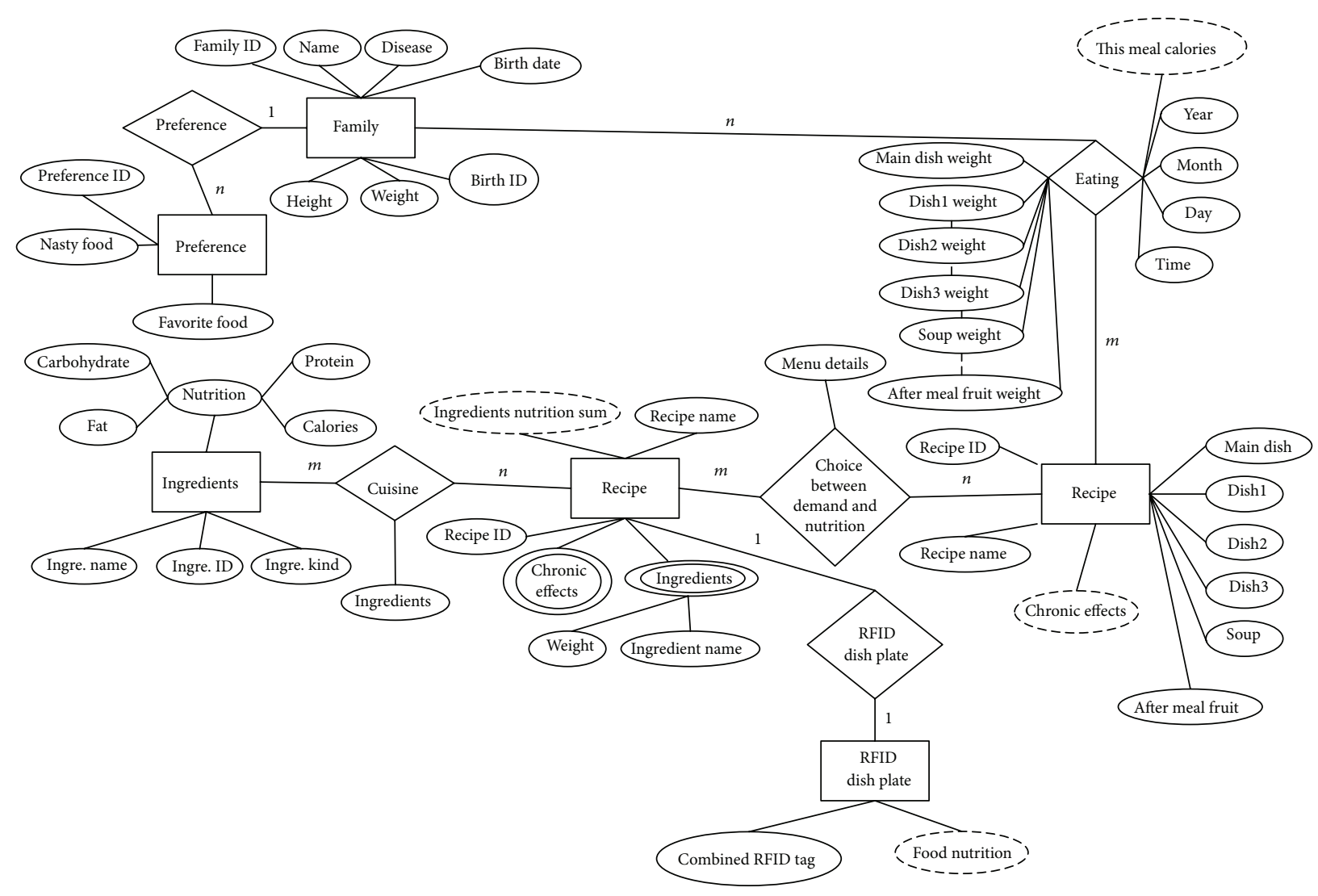

Figure 8: ER-model.

(2) RFID system tray food nutrition: this system is composed of RFID electronic scales and electric plate was used for food weight, food distribution, and nutrition calculation and record. RFID tags are embedded in the chassis plate and each combination plate as the identity of the chassis identification and to record the food nutrition of a set of combination plate sets.

The tables and nutrition plate measuring system of dietary management platform provide basic functions such as add, delete, modify, and query, in which each function is described in detail in the following.

(1) Add: users, through add features, add new basic information for each user, containing name, gender, health status, and so forth, as well as new ingredients, recipes, menus, and personalized menu.

(2) Modify and delete: users, by modifying and deleting functions, modify or delete the added data.

(3) Query: it provides a variety of ways to search for users by name, age, and so forth, and, in the majority of food nutrition, it can also make queries and sorting by kind of food and nutritional ingredients.

(4) RFID tray food nutrition system: this system is a dietary management system integrated with RFID system tray food nutrition and dietary nutrition database. Users can use this system to scale the amount of food ingredients, weigh food weight and nutrition, record daily nutrition, and provide dietary advice.

3.4. System Functions Definition. In this study, the system database uses MS SQL. Based on a user's health status and preferences, it establishes multirecords of a user's preference and nutrition history so that the preferences and nutrients for a single user can be analyzed [19].

To set up a personalized menu for each user, each individually personalized menu offers multirecords selection and modification of daily menu, which is composed of the multirecord menus, recipes, and ingredients. Tables in a dietary database are divided into user basic information, menu composition, analysis of users' nutritional needs, and RFID dishes nutritional data. Each table has a unique primary key, and normalized ER-model of the database is shown in Figure 8.

3.5. System Functions Definition. System environment architecture is constructed using Asp.net, where the main advantage is that users only need to be connected through a web browser platform, without installing additional software, so it allows users to be quickly familiar with operating environment so as to reduce the burden on the job. According to user requirements after finishing the planning, the system 


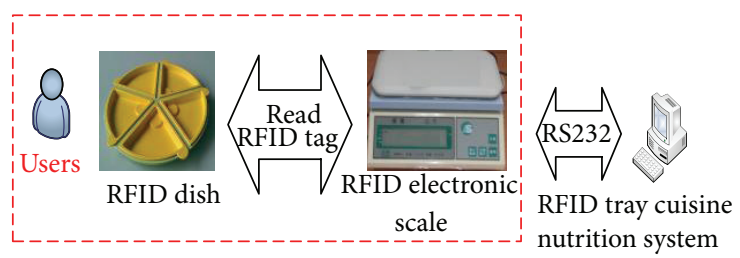

FIgURE 9: System environment architecture diagram.

architecture contains a dietary database, where system environment architecture is shown in Figure 9 [19, 20].

Based on system analysis and planned functions, database is designed and programs are written. The system function framework is outlined in Figure 10.

(1) RFID Dietary Management System. The main device is a plate with embedded RFID tags and RFID tag reading functionality with electronic scales, which makes transmission via RS232 interface with a computer, so users can be authenticated through the RFID plate identification and calculated for his personal dietary.

(2) Dietary Database. Based on the information provided by "Taiwan food nutrition database" through the translation of the calculated weight of the ingredients, it can obtain calories per gram, three major nutrients, dietary fiber, minerals and other nutrients, and minerals, cholesterol, fiber, and other elements. Food nutrition database is established in accordance with the above information to be used for the subsequent cooking dietary ingredients.

\section{Numerical Results}

The system has built thousands of nutrients essential ingredients of foodstuffs. The user can log in to obtain permission to add, delete, and modify the various ingredients of food. The ingredients of food are classified and the system takes one gram as the basic unit of nutritional food ingredients for database, which users can easily access. The result was shown in Figure 11.

The system constructed according to the research objectives and research methods shows results as follows: cooking and catering management system built-in basic ingredients, recipes, menus, and basic analysis with add, delete, and modify functions as shown in Figure 12.

The system will automatically check the basic information of a user to automatically generate the required calorie and three major nutrients required for a day; daily needs of calorie require basal metabolic rate provided by the Department of Health (BMR) formula to calculate the amount of physical activity:

Day calories required $=B M R \times$ activity index.

(1) Basal metabolic rate:

(i) men $\mathrm{BMR}=[13.7 \times$ weight $(\mathrm{kg})]+[5.0 \times$ height $(\mathrm{cm})]-(6.8 \times$ age $)+66$;

(ii) women $\mathrm{BMR}=[9.6 \times$ weight $(\mathrm{kg})]+[1.8 \times$ height $(\mathrm{cm})]-(4.7 \times$ age $)+655$.
(2) Activity index:

(i) sedentary office type $=1.2$ (seldom or no exercise entirely);

(ii) mild activity type $=1.375$ (1-3 times per week doing exercise);

(iii) moderate exercise type $=1.55$ (3-5 times of exercises weekly);

(iv) severe sport $=1.725$ (6-7 times per week doing exercise);

(v) manual type $=1.9$ (heavy exercise or manual workers).

Based on a daily required calorie, the system will automatically calculate three major nutrients:

(1) sugar: $50-60 \%$ of total calories,

(2) protein: $10-20 \%$ of total calories,

(3) fat: not more than $30 \%$ of total calories for the principle.

The system is based on personal food preferences, needs, and physical condition and imports prescription dietary considerations into dietary management system as shown in Figure 13 to easily track and monitor the garnish cooking.

To solve the home diet management system from cooking to garnish with dietary nutrients difficult to control and understanding of issues, this system uses RFID-embedded electronic plate composed of chassis plate and a plurality of subdishes to control each meal intake of nutrients with RFID electronic scales and electronic-control plate. The nutrients of individual subdish servings of dishes obtained are shown in Figure 14. Users do not need to manually update the RFID tag data for each combination dish, and the system automatically records information of each combination plate of food nutrition. After a user simply places his plate on RFID electronic scale, he can very quickly obtain the sum of his own food nutritional intake, as shown in Figure 15 [19, 21].

\section{Discussions}

By using cooking and dietary management system constructed through information technology, the cooked food nutrients can accurately grasp the advantages of information, which can be reused beyond doubt. However, with ingredients, recipes, and menus to be built more and more, if pictures or cooking ingredients cooking methods and steps description can be added, it will make the system interface more user-friendly.

After round electronic dishes are embedded with RFID tags, each subdish without geometric constraints of the chassis can be assembled in any chassis. However, for the people who can only accept customary rectangular plate, it has still room for the electronic meal plate design to be improved in the future.

When RFID electronic plate in the dietary management system performs dish garnish, the amount of food nutrition scale have many variables, such as uneven distribution of food species classification, so the amount of nutrients will be affected. 


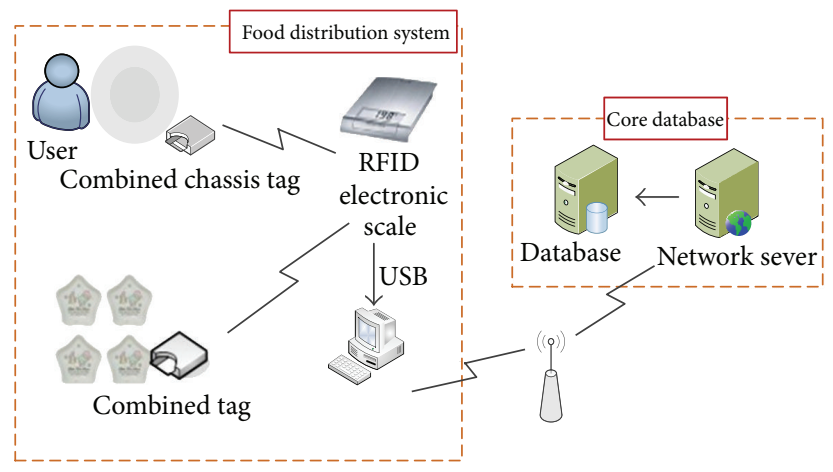

FIgURE 10: RFID dish plate diagram.

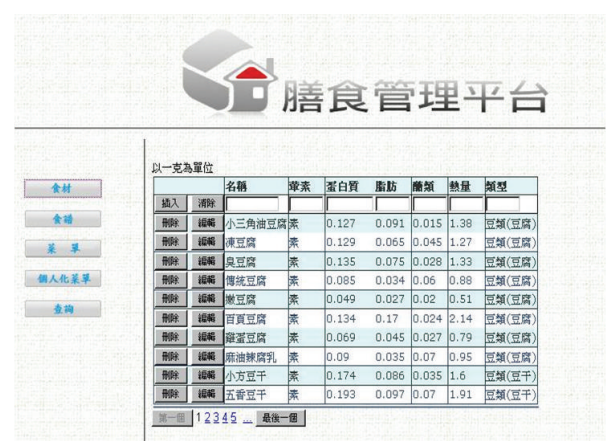

FIGURE 11: System foodstuffs ingredients diagram.

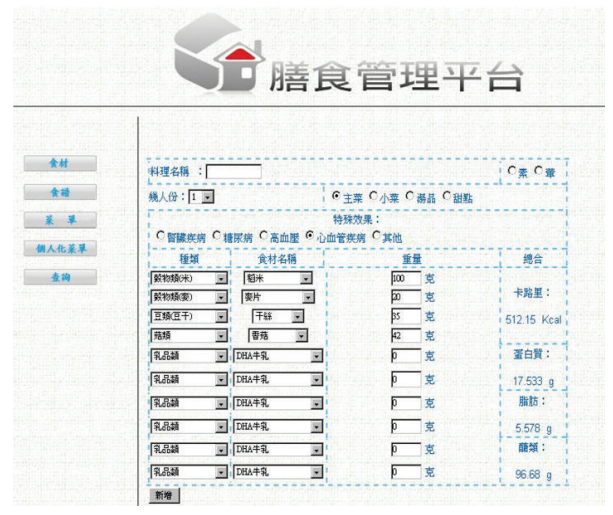

FIGURE 12: System nutritional ingredients diagram.

\section{Conclusions}

When exploring dietary health management research, the widely used approach is to record daily food intake and meal nutrients intake, which is used to assess the user's health and eating habits in order to identify the lack of or excess nutrients.

This study proposed dietary management database and RFID system solutions to improve the following issues.

(1) Dietary database, in addition to providing essential food nutrients, can be used by the user to query using keywords and classification methods so as to quickly

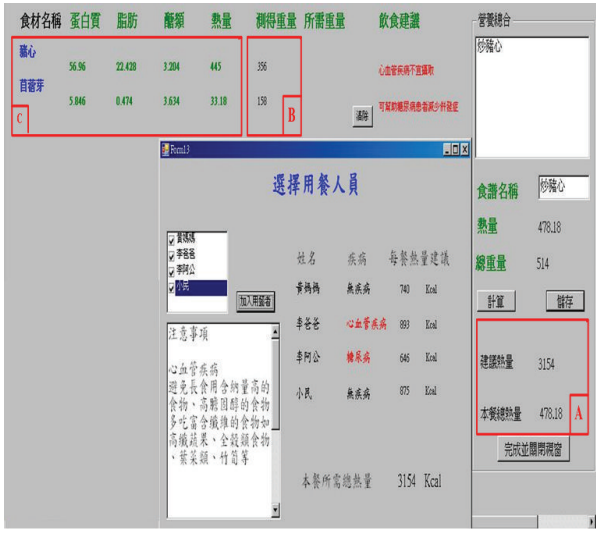

FIGURE 13: System garnish nutritional diagram.

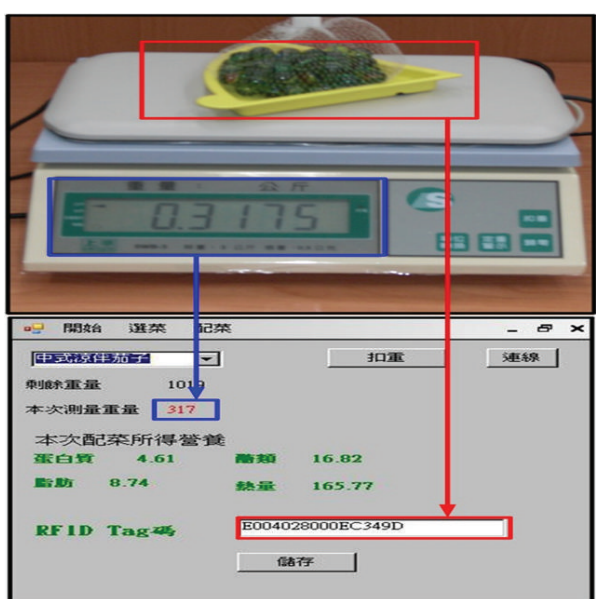

FIGURE 14: Nutritional information of subdish module.

find the sum ingredients for cooking, ingredients, and quick transaction carried out with the add, modify, and delete functions.

(2) The nutritional content of the food is more easily understood by users with grams unit, and the interface for cooking ingredients measurements and cooking configuration is easier to understand. 


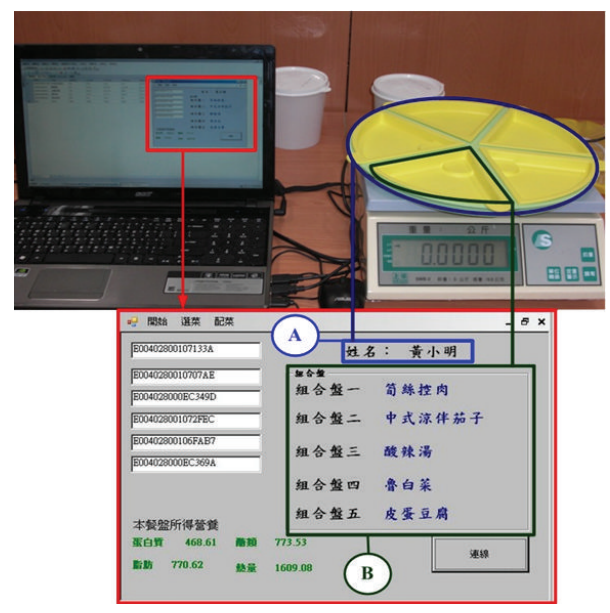

FIGURE 15: Nutritional information of individualized dietary.

(3) RFID dietary management system supported with a cuisine nutrition database provides cooking process of normal home cooking through RFID electronic plate features to record daily dietary intake of nutrients so as to make the dietary management more convenient indeed.

Through this system, to enhance cooking and management accessibility for the individual and the family home, individuals at home can enjoy the same food control services to achieve the purpose of a healthy diet.

\section{Conflict of Interests}

The authors declare that there is no conflict of interests regarding the publication of this paper.

\section{Acknowledgment}

This research was provided by the research-related funding of the National Science Council Research (Project no. NSC1002632-E-252-001-MY3).

\section{References}

[1] A. Bandura, "Self-efficacy: toward a unifying theory of behavioral change," Psychological Review, vol. 84, no. 2, pp. 191-215, 1977.

[2] S. Johnson, "Local area agency on aging supports in-home nutrition education for high-nutritional risk seniors," Journal of Extension, vol. 48, no. 4, 2010.

[3] Department of Health Bureau of Health, Chronic Disease Prevention, 2007, http://health99.hpa.gov.tw/EducZone/edukits .aspx.

[4] Department of Health, Republic Diet Manual, Department of Health, 1994.

[5] Department of Health, Nutritional Status of the Elderly in Taiwan Survey 1999-2000, 2012, http://webpac.lib.tku.edu.tw /lib/item;jsessionid=6FC974AB68A6BBD23EF331737DFD7E11 ?id=chamo: $612010 \&$ theme $=$ tkulib.
[6] J. Salomón, "MyPlate helps remind people how to make smart, healthy eating choices," 2012, http://blog.affinityhealth.org/myplate-helps-remind-people-how-to-make-smart-healthy-eating -choices\#.UuutJ_mSw_p.

[7] T. Vilsack, My Plate, 2012, http://nutrinote.blogspot.tw/.

[8] R. Haigh, “The ageing process: a challenge for design," Applied Ergonomics, vol. 24, no. 1, pp. 9-14, 1993.

[9] F. L. Chung, "Privacy protection and mutual authentication scheme for RFID systems," Journal of e-Business, vol. 10, no. 3, pp. 715-726, 2008.

[10] J. Daemen and V. Rijmen, "Rijndael: the advance encryption standard," Dr. Dobbs Journal, March 2001.

[11] A. L. Xuan, "Exercise and nutrition disorders common in the elderly-physiotherapist perspective," The Gerontologist, vol. 9, no. $1,2005$.

[12] E. K. Kaye, "Bone health and oral health," Journal of the American Dental Association, vol. 138, no. 5, pp. 616-619, 2007.

[13] W. R. Li, RFID applications recorded in the daily diet of home health care [M.S. thesis], The Department of Computer and Communication, Asia University, 2006.

[14] G. H. Chang, Diet sensing table-the wisdom of eating behavior sensing plane [M.S. thesis], Institute of Information Engineering, National Taiwan University, 2006.

[15] L. X. Yao and M. Y. Wang, To use the principles of design and explore personal diet management system interface, [Master thesis], Department of Industrial Engineering and Engineering Management, National Tsing Hua University, 2011.

[16] T. Miyazaki, G. C. De Silva, and K. Aizawa, "Image-based calorie content estimation for dietary assessment," in Proceedings of the 13th IEEE International Symposium on Multimedia (ISM '11), pp. 363-368, December 2011.

[17] G. Kovásznai, "Developing an expert system for diet recommendation," in Proceedings of the 6th IEEE International Symposium on Applied Computational Intelligence and Informatics (SACI '11), pp. 505-509, Timişoara, Romania, May 2011.

[18] A. Mariappan, M. Bosch, F. Zhu et al., "Personal dietary assessment using mobile devices," in Computational Imaging VII, vol. 7246 of Proceedings of SPIE, San Jose, Calif, USA, 2009.

[19] Y. H. Liang, P. H. Chen, and J. J. Chang, "RFID technology to construct cooking and meal management system," in Proceedings of the 4th Medical Digital Learning Seminars, Chiayi, Taiwan, September 2013.

[20] L. H. Lichtenstein, H. Rasmussen, W. W. Yu, S. R. Epstein, and R. M. Russell, "Modified MyPyramid for older adults," Journal of Nutrition, vol. 138, no. 1, pp. 5-11, 2008.

[21] Y. H. Liang, P. H. Chen, and J. J. Chang, "Integrating RFID technology and dietary management of electronic plate," in Proceedings of the Digital Life Science and Technology Symposium, pp. 245-250, Yunlin, Taiwan, August 2012. 


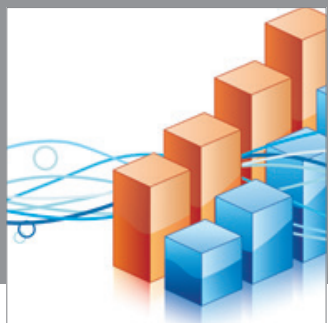

Advances in

Operations Research

mansans

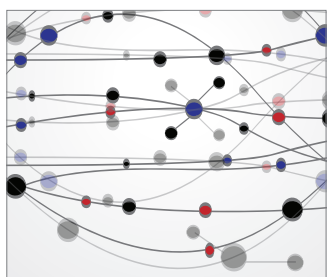

The Scientific World Journal
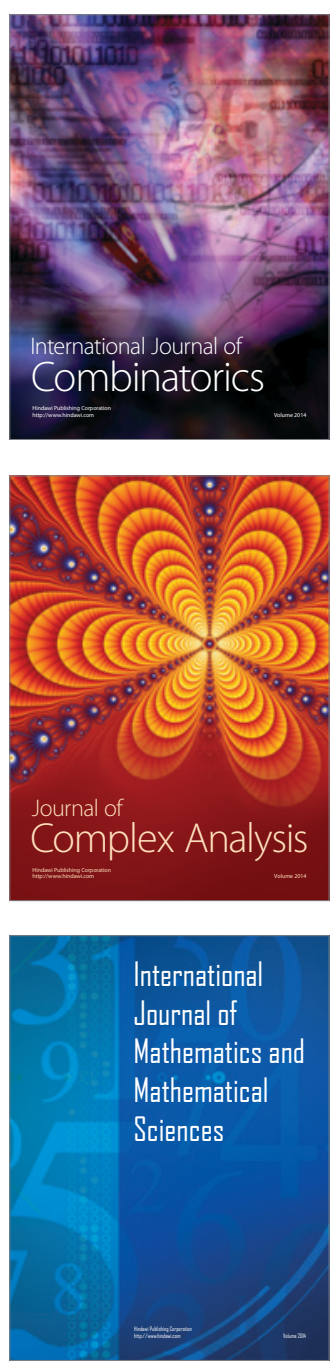
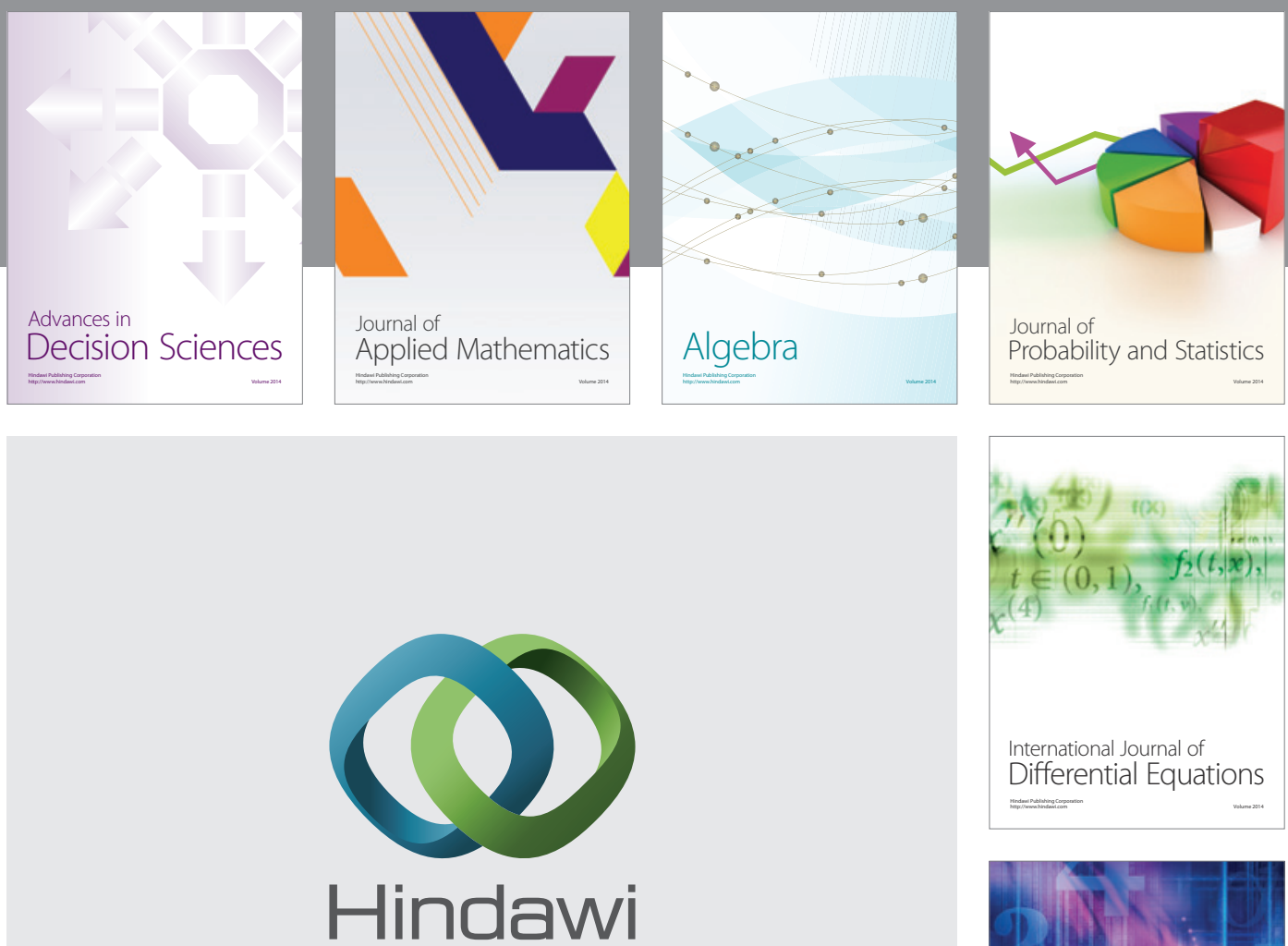

Submit your manuscripts at http://www.hindawi.com
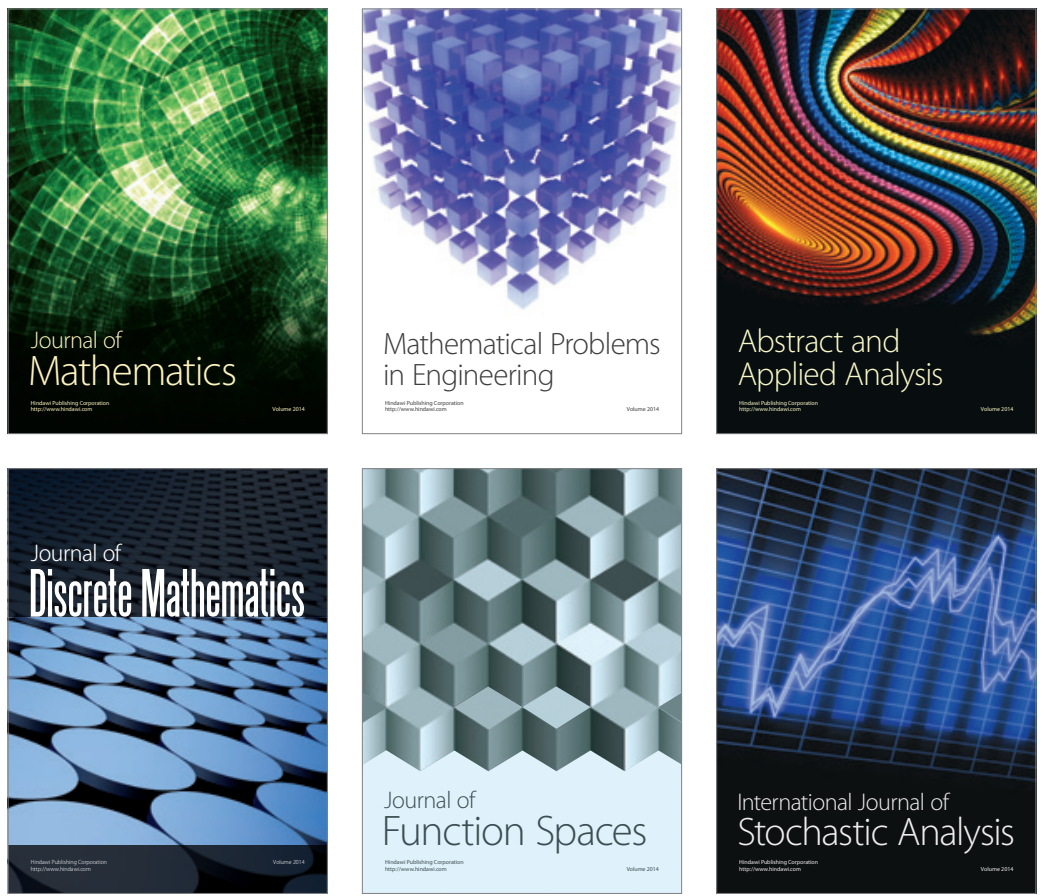

Journal of

Function Spaces

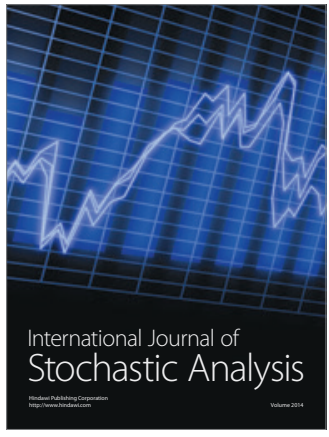

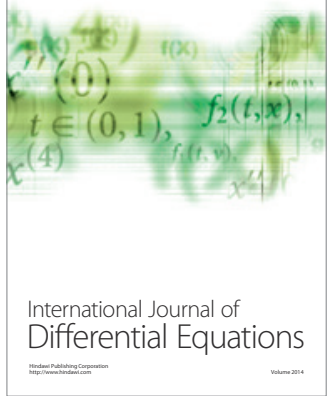
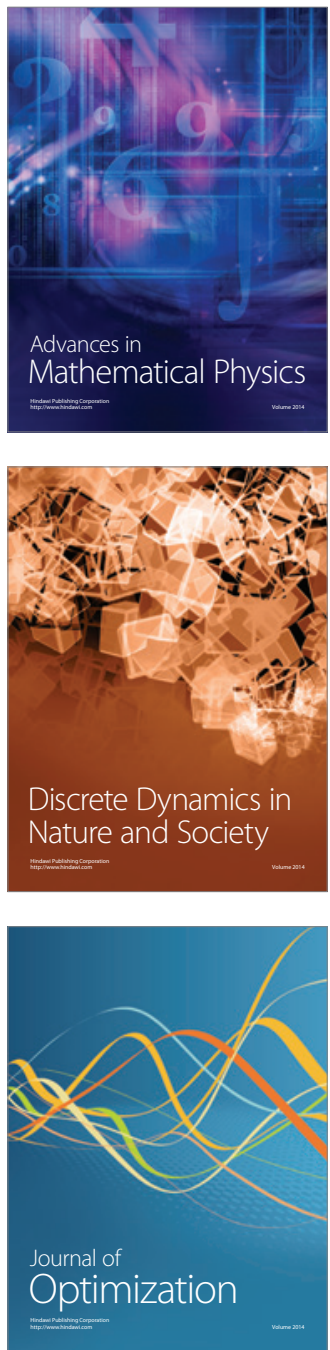